\title{
Editorial: CRISPR-Cas in Agriculture: Opportunities and Challenges
}

\author{
Sandeep Kumar ${ }^{1 \dagger}$, Linda Ann Rymarquis ${ }^{2 \dagger}$, Hiroshi Ezura ${ }^{3,4 \dagger}$ and Vladimir Nekrasov ${ }^{5 * \dagger}$ \\ ${ }^{1}$ Corteva Agriscience, Johnston, IA, United States, ${ }^{2}$ Bayer Crop Science, Chesterfield, MO, United States, ${ }^{3}$ Faculty of Life \\ and Environmental Sciences, University of Tsukuba, Tsukuba, Japan, ${ }^{4}$ Tsukuba Plant Innovation Research Center (T-PIRC), \\ University of Tsukuba, Tsukuba, Japan, ${ }^{5}$ Plant Sciences Department, Rothamsted Research, Harpenden, United Kingdom
}

Keywords: agriculture, genome editing, crop, CRISPR, Cas9, Cas12a, policy, regulation

Editorial on the Research Topic

CRISPR-Cas in Agriculture: Opportunities and Challenges

\section{OPEN ACCESS}

Edited and reviewed by:

Raúl Alvarez-Venegas,

Centro de Investigaciones y Estudios

Avanzados, Instituto Politécnico

Nacional de México

(CINVESTAV), Mexico

*Correspondence:

Vladimir Nekrasov

vladimir.nekrasov@rothamsted.ac.uk

tORCID:

Sandeep Kumar

orcid.org/0000-0001-8614-9700

Linda Ann Rymarquis

orcid.org/0000-0003-0297-8371

Hiroshi Ezura

orcid.org/0000-0003-1443-2210

Vladimir Nekrasov

orcid.org/0000-0001-9386-1683

Specialty section:

This article was submitted to

Plant Biotechnology,

a section of the journal

Frontiers in Plant Science

Received: 25 February 2021

Accepted: 02 March 2021

Published: 26 March 2021

Citation:

Kumar S, Rymarquis LA, Ezura $\mathrm{H}$ and Nekrasov V (2021) Editorial: CRISPR-Cas in Agriculture:

Opportunities and Challenges.

Front. Plant Sci. 12:672329.

doi: 10.3389/fpls.2021.672329

\section{CRISPR-CAS TECHNOLOGY: STATE OF THE ART, POLICY, AND REGULATION}

CRISPR-Cas genome editing technology is developing at a rapid pace and new molecular tools, such as CRISPR nucleases, are becoming regularly available. As part of this Research Topic, Bandyopadhyay et al. provide a comprehensive overview of Cas12a, a CRISPR nuclease formerly known as Cpf1. In their review article, the authors cover structural and mechanistic aspects of Cas12a in comparison with Cas9, the most commonly used CRISPR nuclease. They also highlight uses of Cas12a for the purpose of improving agriculturally important traits in various crops. An overview of Cas9 genome editing applications in plants is provided by El-Mounadi et al. who introduce the reader to the mechanism of Cas 9 activity, methods of its delivery to plant cells (i.e., transformation techniques), give examples of improving crop traits using CRISPR-Cas9, and touch on biosafety and regulatory aspects associated with genome editing. A number of countries (e.g., the USA, Brazil, Argentina, and Japan) have already exempted genome edited crops, which do not carry transgenic DNA or novel combination of genetic material (i.e., not similarly achievable through conventional breeding), from being regulated similarly to Genetically Modified Organisms (GMOs) as genetically engineered (GE) organisms (Schmidt et al., 2020). Although the abovementioned countries have passed legislation allowing cultivation of genome edited crops without GE regulation, the public dialogue and policy developments on the issue are evolving. In the case of Japan, Tabei et al. analyze Twitter conversation on genome-edited foods and their labeling over the period from May to October 2019. The analysis reveals that $54.5 \%$ of relevant tweets were statements opposed to food produced using genome edited crops, while only $7 \%$ were statements in favor of it. The remaining $38.5 \%$ of tweets were statements deemed neutral. Although the analysis was not necessarily representative of the wider Japanese society due to bias among Twitter users, the study underlines the importance of a continuous public dialogue on the issue of genome edited crops in Japan and the rest of the world.

\section{CRISPR-CAS AS A TOOL FOR GENE FUNCTION STUDIES AND CROP TRAIT IMPROVEMENT}

One of the factors impacting the efficiency of CRISPR-Cas is the expression level of the gene encoding the respective nuclease during different developmental stages of the plant. For example, 
CRISPR-Cas mutagenesis in Arabidopsis often results in chimerism in the T1 generation due to low expression of Cas 9 (when driven by a promoter, such as 35S) during the zygote and early embryo developmental stages (Feng et al., 2014). To address the chimerism problem, egg cell-specific promoters could be a good choice to drive CRISPR nuclease gene expression for increasing the rates of CRISPR-Cas-induced germline mutations, which are inherited by the next generation (Wang et al., 2015; Yan et al., 2015; Mao et al., 2016). Zheng et al. test four different egg cell-specific promoters (two from Arabidopsis and two from soybean) to drive expression of Cas9 in Arabidopsis and soybean transgenic lines. Out of the four promoters, AtEC1.2e1.1p, which is an adaptation of the previously published AtEC1.1 and AtEC1.2 promoter fusion (Wang et al., 2015), seems to perform best in both plant species. The study by Zheng et al. therefore contributes new molecular tools for efficient targeted mutagenesis in a model plant, such as Arabidopsis, and an economically important crop, such as soybean.

Virdi et al. and Zhang et al. highlight the use the CRISPRCas for gene function analysis. Virdi et al. studied the soybean KASI gene, which is crucial for conversion of sucrose to oil. They demonstrate that CRISPR-Cas induced knockout and inframe deletion of GmkasI alleles, have an increase in seed sucrose content and a decrease in total seed oil content relative to wild type. These phenotypes are consistent with what was observed in the mutant line where the GmKASI gene is disrupted by a reciprocal chromosomal translocation. Thus, the authors prove that the phenotype of the line carrying the chromosomal translocation is indeed due to disruption of the GmKASI gene.

Zhang et al. report on knocking out 63 genes involved in immune response in tomato. The authors performed a detailed analysis of the types of mutations generated at an average frequency of $68 \%$, which is similar to previously reported rates for CRISPR-Cas mutagenesis in tomato (Brooks et al., 2014; Nekrasov et al., 2017). They demonstrate that the mutations were transmitted through the germline to the next generation. The off-target analysis they performed for 12 guide RNAs showed no mutations at off-target sites with up to four mismatches and, indicating the high precision of CRISPR-Cas in tomato, this was consistent with what was previously reported (Nekrasov et al., 2017; Hahn and Nekrasov, 2019). The knockout lines are cataloged in the online Plant Genome Editing Database (PGED; http://plantcrispr.org; Zheng et al., 2019).

The CRISPR-Cas technology is a versatile genome editing tool that has been used to improve agriculturally important crop traits, such as quality, disease resistance, and herbicide tolerance. In potato, enzymatic browning is a serious problem for both growers and the industry as it decreases the quality of both the fresh and processed product. González et al. report on a successful application of CRISPR ribonucleoproteins for the purpose of reducing enzymatic browning in potato tubers by targeting the Polyphenol Oxidase 2 gene (StPPO2), one of the five potato PPO genes. By disrupting all four copies of StPPO2 the authors achieved a dramatic reduction in tuber PPO activity (up to 69\%) and enzymatic browning (73\%). The findings presented by González et al. are consistent with the reported reduction in potato browning achieved by silencing the StPPO2 gene using RNAi (Richael, 2021).

In addition to dicot crops, CRISPR-Cas has been extensively used for trait improvement in cereals, such as rice and maize. As an example, Zafar et al. are reporting on enhancing disease resistance to Xanthomonas oryzae pv. oryzae (Xoo), a pathogen causing bacterial blight in rice, by editing the promoter of a susceptibility (S) gene. Xoo secretes transcription activatorlike effectors (TALEs) that activate host $S$ genes, such as OsSWEET family members. Using CRISPR-Cas, the authors introduced deletions overlapping with effector binding elements (EBEs) recognized by AvrXa7/PthXo3 or TalF TALEs within the promoter of the OsSWEET14 gene in the Super Basmati elite cultivar. Mutant rice lines carrying deletions in the AvrXa7/PthXo3 EBEs showed enhanced resistance to the Xoo strain carrying $A v r X a 7$ in agreement with previously published reports (Li et al., 2012; Blanvillain-Baufumé et al., 2017; Oliva et al., 2019; Xu et al., 2019).

In another report, Komatsu et al. address the problem of "volunteer rice" that emerges from seeds falling into fields during the harvest season and then spontaneously germinates the next spring. If volunteer rice originates from a feed variety, it can compromise the quality of rice meant for human consumption, which is grown during the next season. As many japonica rice cultivars are resistant to beta-triketone herbicides (bTH), such as benzobicyclon (BBC), the authors tested the feasibility of engineering $\mathrm{BBC}$ susceptibility in japonica rice (cv. Nipponbare) by targeting the HIS1 gene using the cytosine base editor (CBE). They successfully generated a number of his1 knockout lines by eliminating the start codon or introducing premature stop codons within the HIS1 coding sequence. The his1 loss-offunction lines appear to be susceptible to BBC and other betatriketone pesticides, paving a way to controlling volunteer rice in the field by applying the same strategy to BBC-resistant feed rice cultivars.

In maize, Gao et al. report on a CRISPR-Cas9- and recombinase-mediated strategy for stacking biotech traits within complex trait loci (CTLs). Each CTL spans 4-5 cM and includes 12-30 pre-selected sites used for insertion of a landing pad via homology-directed repair (HDR) using CRISPR-Cas9. As a result, the authors generated a set of individual transgenic lines, each carrying a landing pad at one of the preselected sites within one of the four CTLs. At the following step, the landing padcarrying lines were used for integration of trait genes using the FLP recombinase. Finally, integrated trait genes were stacked on the same chromosome by crossing respective individual transgenic lines and selecting recombinants. The study by Gao et al. therefore presents a modular and flexible way of stacking biotech traits, as compared to previously reported strategies involving recombinases or zinc finger/homing endonucleases (Ow, 2011; D'Halluin et al., 2013; Kumar et al., 2015), due to the possibility for trait genes to be easily combined or separated (e.g., in case one of them loses efficiency) by conventional breeding.

This volume highlights the many opportunities that the CRISPR-Cas systems hold for Agriculture. Both Cas9 and Cas12a have been proven to drive edits in plants and new improvements, such as using germline-specific 
promoters that increase heritability, will only enhance their potential (Zheng et al.). The CRISPR-Cas systems have been successfully implemented to forward basic research, such as gene discovery of oil (Virdi et al.) and disease genes (Zhang et al.), as well as improve agricultural outcomes e.g., via decreasing potato browning (González et al.), improving disease resistance (Zafar et al.), mitigating volunteer rice (Komatsu et al.), and stacking biotech traits (Gao et al.). In order for this potential to be fully realized, Tabei et al. showed that work must be done to gain public acceptance and ensure implementation of favorable public policy.

\section{REFERENCES}

Blanvillain-Baufumé, S., Reschke, M., Solé, M., Auguy, F., Doucoure, H., Szurek, B., et al. (2017). Targeted promoter editing for rice resistance to Xanthomonas oryzae pv. oryzae reveals differential activities for SWEET14-inducing TAL effectors. Plant Biotechnol. J. 15, 306-317. doi: 10.1111/pbi.12613

Brooks, C., Nekrasov, V., Lippman, Z. B., and Van Eck, J. (2014). Efficient gene editing in tomato in the first generation using the clustered regularly interspaced short palindromic repeats/CRISPR-Associated9 System. Plant Physiol. 166, 1292-1297. doi: 10.1104/pp.114.247577

D’Halluin, K., Vanderstraeten, C., Hulle, J. V., Rosolowska, J., Brande, I. V. D., Pennewaert, A., et al. (2013). Targeted molecular trait stacking in cotton through targeted double-strand break induction. Plant Biotechnol. J. 11, 933-941. doi: 10.1111/pbi.12085

Feng, Z., Mao, Y., Xu, N., Zhang, B., Wei, P., Yang, D.-L., et al. (2014). Multigeneration analysis reveals the inheritance, specificity, and patterns of CRISPR/Cas-induced gene modifications in Arabidopsis. Proc. Natl. Acad. Sci. U.S.A. 111, 4632-4637. doi: 10.1073/pnas.1400822111

Hahn, F., and Nekrasov, V. (2019). CRISPR/Cas precision: do we need to worry about off-targeting in plants? Plant Cell Rep. 38, 437-441. doi: 10.1007/s00299-018-2355-9

Kumar, S., AlAbed, D., Worden, A., Novak, S., Wu, H., Ausmus, C., et al. (2015). A modular gene targeting system for sequential transgene stacking in plants. J. Biotechnol. 207, 12-20. doi: 10.1016/j.jbiotec.2015.04.006

Li, T., Liu, B., Spalding, M. H., Weeks, D. P., and Yang, B. (2012). High-efficiency TALEN-based gene editing produces disease-resistant rice. Nat. Biotechnol. 30, 390-392. doi: 10.1038/nbt.2199

Mao, Y., Zhang, Z., Feng, Z., Wei, P., Zhang, H., Botella, J. R., et al. (2016). Development of germ-line-specific CRISPR-Cas9 systems to improve the production of heritable gene modifications in Arabidopsis. Plant Biotechnol. J. 14, 519-532. doi: 10.1111/pbi.12468

Nekrasov, V., Wang, C., Win, J., Lanz, C., Weigel, D., and Kamoun, S. (2017). Rapid generation of a transgene-free powdery mildew resistant tomato by genome deletion. Sci. Rep. 7:482. doi: 10.1038/s41598-017-00578-x

Oliva, R., Ji, C., Atienza-Grande, G., Huguet-Tapia, J. C., Perez-Quintero, A., $\mathrm{Li}, \mathrm{T}$., et al. (2019). Broad-spectrum resistance to bacterial blight in rice using genome editing. Nat. Biotechnol. 37, 1344-1350. doi: 10.1038/s41587-01 9-0267-z

\section{AUTHOR CONTRIBUTIONS}

VN wrote the paper. SK, LR, and HE made direct contributions to the work, reviewed, and approved it for publication. All authors contributed to the article and approved the submitted version.

\section{ACKNOWLEDGMENTS}

VN receives grant-aided support from the Biotechnology and Biological Sciences Research Council (BBSRC) Designing Future Wheat (DFW) programme (grant number BBS/E/C/000I0220) and Newton Fund (grant number BB/R019827/1).

Ow, D. W. (2011). Recombinase-mediated gene stacking as a transformation operating system. J. Integr. Plant Biol. 53, 512-519. doi: 10.1111/j.1744-7909.2011.01061.x

Richael, C. M. (2021). "Development of the Genetically Modified Innate ${ }^{\circledR}$ Potato," in Plant Breeding Reviews, ed I. Goldman (Hoboken, NJ: John Wiley \& Sons, Ltd.), 57-78. doi: 10.1002/9781119717003.ch3

Schmidt, S. M., Belisle, M., and Frommer, W. B. (2020). The evolving landscape around genome editing in agriculture. EMBO Rep. 21:e50680. doi: 10.15252/embr.202050680

Wang, Z.-P., Xing, H.-L., Dong, L., Zhang, H.-Y., Han, C.-Y., Wang, X.-C., et al. (2015). Egg cell-specific promoter-controlled CRISPR/Cas9 efficiently generates homozygous mutants for multiple target genes in Arabidopsis in a single generation. Genome Biol. 16:144. doi: 10.1186/s13059-015-0715-0

Xu, Z., Xu, X., Gong, Q., Li, Z., Li, Y., Wang, S., et al. (2019). Engineering broadspectrum bacterial blight resistance by simultaneously disrupting variable TALE-binding elements of multiple susceptibility genes in rice. Mol. Plant 12, 1434-1446. doi: 10.1016/j.molp.2019.08.006

Yan, L., Wei, S., Wu, Y., Hu, R., Li, H., Yang, W., et al. (2015). High-efficiency genome editing in Arabidopsis using YAO promoter-driven CRISPR/Cas9 system. Mol. Plant 8, 1820-1823. doi: 10.1016/j.molp.2015.10.004

Zheng, Y., Zhang, N., Martin, G. B., and Fei, Z. (2019). Plant genome editing database (PGED): a call for submission of information about genome-edited plant mutants. Mol. Plant 12, 127-129. doi: 10.1016/j.molp.2019.01.001

Conflict of Interest: SK is an employee of Corteva Agriscience ${ }^{\mathrm{TM}}$. LR is an employee of Bayer.

The remaining authors declare that the research was conducted in the absence of any commercial or financial relationships that could be construed as a potential conflict of interest.

Copyright (c) 2021 Kumar, Rymarquis, Ezura and Nekrasov. This is an open-access article distributed under the terms of the Creative Commons Attribution License (CC $B Y)$. The use, distribution or reproduction in other forums is permitted, provided the original author(s) and the copyright owner(s) are credited and that the original publication in this journal is cited, in accordance with accepted academic practice. No use, distribution or reproduction is permitted which does not comply with these terms. 